\title{
Multiple defect interpretation based on Gaussian processes for MFL technology
}

\author{
Buddhi Wijerathna ${ }^{a}$, Teresa Vidal-Calleja ${ }^{a}$, Sarath Kodagoda ${ }^{a}$, \\ Qiang Zhang ${ }^{a}$ and Jaime Valls Miro ${ }^{a}$ \\ ${ }^{a}$ Centre for Autonomous Systems, Faculty of Engineering and IT, \\ University of Technology, Sydney, NSW 2007, Australia.
}

\begin{abstract}
Magnetic Flux Leakage (MFL) technology has been used in non-destructive testing for more than three decades. There have been several publications in detecting and sizing defects on metal pipes using machine learning techniques. Most of these literature focus on isolated defects, which is far from the real scenario.

This study is towards the generalization of interpretation of the leakage flux in the presence of multiple defects based on simulation models, together with data-driven inference methodologies, such as Gaussian Process (GP) models. A MFL device has been simulated using both COMSOL Multiphysics and ANSYS software followed by prototyping the same device for experimental validations.

Multiple defects with different geometrical configurations were introduced on a cast iron pipe sample and both radial and axial components of the leakage field have been measured. It was observed that both axial and radial components differ with different defect configurations. We propose to use GP to solve the inverse model problem by capturing such behaviors, i.e. to recover the profile of a cluster of defects from the measurements of a MFL device. The data was used to learn the non-parametric GP model with squared exponential covariance function and automatic relevance determination to solve this regression problem.

Extensive quantitative and qualitative evaluations are presented using simulated and experimental data that validate the success of the proposed non-parametric methodology for interpreting the profiling of clusters of defects with MFL technology.
\end{abstract}

Keywords: Nondestructive testing, Magnetic flux leakage, Finite element analysis, Data driven inference, Gaussian processes.

\section{INTRODUCTION}

Modern industrial asset maintenance requires reliable and accurate inspection techniques. Non-destructive evaluation (NDE) techniques are commonly used for infrastructure inspection, for instance to assess physical defects and anomalies without permanently altering the asset. With the current advancements of the NDE technology, in service inspection is getting more popular in industries such as water, petrochemical, transportation, energy and metal.

Magnetic Flux Leakage (MFL) technology has been used in NDE for more than three decades. In the MFL inspection process, a sample of a ferromagnetic material is magnetized using a strong magnetic field. In the presence of defects or anomalies, a magnetic flux "leak" can be detected. Suitable sensors are employed to scan this leakage field on which different signal processing techniques are applied for detection and sizing of them. The use of advanced signal processing and analysis technologies may result in more accurate modeling and prediction. In particular, machine learning algorithms appear well-suited for robust modelling of MFL signals.

Two main frameworks are generally employed to model MFL signals. The more traditional forward approach requires knowledge of precise calibration samples to generate MFL signals which are compared with fresh measurements to infer the most likely defect profile. The inverse approach fits a model that can be used to predict the defect configuration from the MFL signals. This study is focused on the inverse model of MFL signals by using state of the art machine learning techniques in order to detect and size multiple defects.

Further author information: (Send correspondence to B. Wijerathna)

E-mail: buddhi.s.wijerathna@student.uts.edu.au, Telephone: +61 295143148

Nondestructive Characterization for Composite Materials, Aerospace Engineering, Civil Infrastructure, and Homeland Security 2013, edited by Tzu Yang Yu, Andrew L. Gyekenyesi, Peter J. Shull, Aaron A. Diaz, H. Felix Wu, Proc. of SPIE Vol. 8694, 86941Z - (C) 2013 SPIE - CCC code: 0277-786X/13/\$18 - doi: 10.1117/12.2009966 
Supervised learning in the form of regression is an important constituent of machine learning for inferring a function from a "labeled" data set, e.g. measurements associated with their ground truth. Traditionally parametric models have been used for this purpose. These models have a possible advantage in ease of interpretability, but for complex data sets, simple parametric models may lack expressive power, and their more complex counterparts may not be easy to work with in practice. The advent of kernel machines, such as Gaussian Processes ${ }^{1}$ (GP) has opened the possibility of flexible models which are practical to work with. Gaussian Process models can conveniently be used to formulate a Bayesian framework for nonlinear regression. In this paper we will use GP models to solve the inverse MFL model problem.

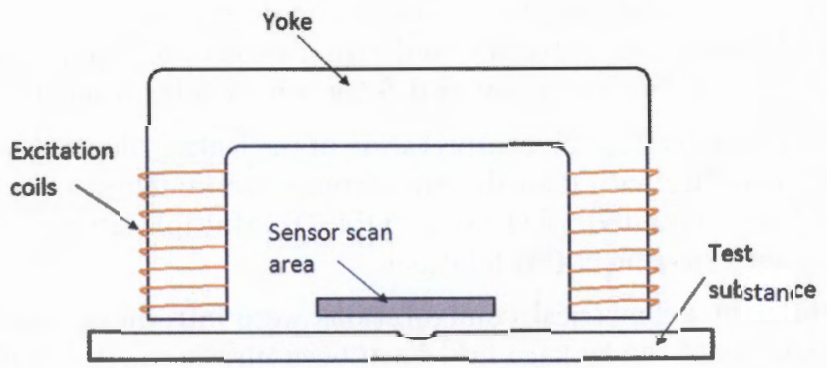

Figure 1: Illustration of a generic MFL device.

Gaussian process models, as any other supervised learning method, require a vast number of examples to accurately model the data. It is not practical to manually acquire a data set that encompasses the range of conditions (multiple defect configurations) which may be expected in practice. Thus realistic numerical simulations such as Finite Element Analysis (FEA) are well-motivated to produce the variability required. In this paper, we validated the accuracy of our simulation models comparing, not only between two different simulation software (COMSOL Multiphysics and ANSYS), but also with the output of an experimental setup that is used for evaluation. Figure 1 shows a diagram of the basic MFL experimental setup.

The rest of the paper is organized as follows. Section 2 presents the literature review. In Section 3 an overview of the approach is described. MFL description together with the simulation models are presented in Section 4. Section 5 describes the dimensionality reduction method based on feature extraction. A detailed explanation of the regression algorithm based on Gaussian process model is presented in Section 6. Finally results and conclusion are presented in Sections 7 and 8 respectively.

\section{LITERATURE REVIEW}

Modelling and analyzing magnetic flux leakage has been a focus of the researchers for more than three decades. Zatsepin and Shcherbinin ${ }^{2}$ modelled the leakage field from infinitely long surface-breaking cracks considering a point dipole, linear dipole or strip dipole in terms of magnetic charges induced by the external magnetic field on the bounding surfaces of the cracks. Later Shcherbinin and Pashagin ${ }^{3}$ developed a more sophisticated model extending the previous work into cracks with finite dimensions. This study was the basis for most of the work published later on the interaction between the defects and the leakage field.

Forster ${ }^{4}$ described mathematically the magnetic flux leakage phenomenon. He claimed that for a better understanding of the magnetic flux leakage phenomenon the determination of the magnetic field strength inside the defect as a function of defect geometry, the magnetic properties of the material, and the applied field strength are essential. Minkov ${ }^{5}$ applied the dipolar model to single flows of irregular cross sections by considering the defect filled with magnetic dipoles aligned parallel to the excitation field, each dipole having a strength directly proportional to the defect depth. They proposed a method for estimating the sizes of surface cracks in magnetic materials based on applying a magnetic field, determining the leakage magnetic field in the vicinity of a crack by moving a Hall element along one or two scanning lines crossing the crack on the surface of the material, and measuring the corresponding Hall voltage distribution. 
For the best of our knowledge, most of the published work up to date on dipolar modelling and sizing discusses isolated defects with few exceptions such as Uetake and Saito ${ }^{6}$ and Mandache. ${ }^{7}$ The first study is limited to slots with parallel walls of maximum of $4 \mathrm{~mm}$ in length, whereas the second is more focused on developing an analytical solution for the inverse problem of specific type of defects. In this paper, we focus on interpreting general types of multiple defects using a supervised learning technique.

With the rapid advancement of the computational capabilities, machine learning techniques are now being used in solving the inverse MFL problem. Artificial neural networks (ANN) are commonly used in modelling complex relationships between inputs and outputs or to find patterns in data. A.A. Carvalho ${ }^{8}$ evaluates the use of ANN for pattern recognition of MFL signals in weld joints. Pradeep Ramuhalli ${ }^{9}$ proposed the use of ANN as forward models to determine defect parameters such as the defect length, depth, and shape (profile) from the measured values of the flux density B. In this paper, we opt to use Gaussian process regression, ${ }^{1}$ which will inherently give a measure of certainty in the recovered defect profile.

\section{APPROACH OVERVIEW}

Inverse modelling is a general framework that is used to infer information about a physical system that we are interested in from observed measurements. In particular, our approach consists in learning the inverse model to characterise the geometry of multiple defects, i.e. depth, width, separation and number of defects in a metal piece using MFL measurements as inputs. We make use of simulated and real data with its corresponding defect dimensions to build these models in a supervised learning manner.

Let us consider the modeling process to estimate for instance the depth of the defects from the MFL device measurements. Let $\left\{\left(\mathbf{w}_{0}, v_{0}\right),\left(\mathbf{w}_{1}, v_{1}\right), \ldots,\left(\mathbf{w}_{N}, v_{N}\right)\right\}$ denote a data set of $N$ hall sensor readings associated with its corresponding depth (i.e. the ground truth), where $v_{i} \in V$ represents the depth of a defect and $\mathbf{w}_{i} \in \Re^{D}$ the noisy hall sensor readings. The dimensionality of $\mathbf{w}_{i}$ is in general high (being all the punctual hall senor measurements in the horizontal axis extracted from the 2-D sensor array); therefore, a first step is to reduce the dimensionality of $\mathbf{w}_{i}$ through a feature extraction process to produce a set of low-dimensional signals with its corresponding depth, $\Psi=\left\{\left(\mathbf{u}_{0}, v_{0}\right),\left(\mathbf{u}_{1}, v_{1}\right), \ldots,\left(\mathbf{u}_{N}, v_{N}\right)\right\}, \mathbf{u}_{i} \in \Re^{d}, d<<D$. The density $P(v \mid \mathbf{u})$ must then be estimated from $\Psi$, without the assumption that $\Psi$ is uniformly distributed over $V .{ }^{10}$

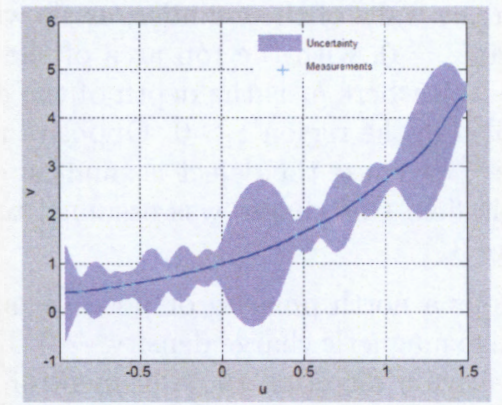

Figure 2: Illustration of Gaussian processes modeling task.

Figure 2 illustrates an 1-D example where $v$ axis shows the state-space, while the $u$-axis shows the feature space: the space of 1-D signals extracted from the sensor readings. The solid line shows the most likely sensor reading for each defect depth. When evaluating the likelihood of a particular sensor reading, there are two sources of uncertainty that must be accounted for: the uncertainty in the depth of the defect and the noise of the sensor. In Figure 2, sensor noise dominates in the densely sampled area, while depth uncertainty dominates near the edges and in the middle of the state-space.

Given this model, it is straightforward to infer depth of the defects for any MFL device reading. In a similar manner, the models to infer other defect dimensions such as width, separation or the number of defects in a given MFL reading can be learned. In this paper, we will consider these dimensions independent from each other to learn independent models which estimate each quantity separately as shown in Section 6. 


\section{MAGNETIC FLUX LEAKAGE}

When the excitation magnetic field is applied to a test substance, a localized corrosion pit acts as a region of high magnetic reluctance. Therefore most of the flux lines are diverted around the defect, creating a dipole effect on the defect, resulting a leakage magnetic field. In this study, it is assumed that a Dipolar magnetic charge (DMC) is developed on the defect faces intersecting the magnetic field as a result of its interaction with the excitation field following the previous work published on DMC by Edwards and Palmer, ${ }^{11}$ Catalin Mandache and Lynann Clapham. ${ }^{7}$ Moreover, local variations in magnetization and permeability were avoided by assuming high magnetic excitation, corresponding to the saturation region of the material.

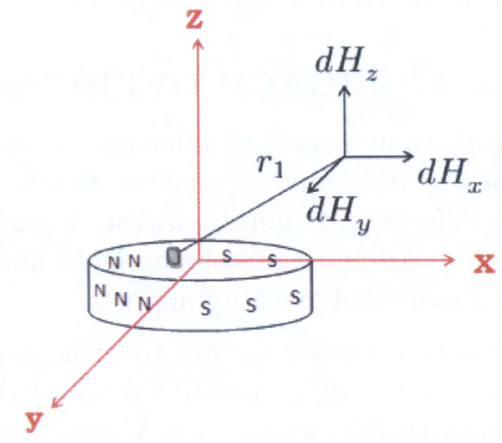

Figure 3: Dipolar magnetic charge models for a cylindrical defect.

Let us consider a cylindrical defect with its longitudinal axis (z-direction) perpendicular to the applied magnetic field (x-direction), ${ }^{7}$ as schematically represented in Figure 3 . The defect is assumed to be milled on the ferromagnetic material and open to the atmospheric air. The magnetic field lines diverge around the low permeability flaw, inducing a DMC on the walls of the cylinder, as shown in the figure. The surface of the test substance is defined by the $x y$ plane at $z=0$, with the top area of the cylindrical defect centered at $(0,0,0)$, and its bottom area centered at $\left(0,0,-b_{1}\right)$, where $b_{1}$ is the depth of the defect. The MFL signal induced by the type of defect just described was sampled in the region $z>0$. Opposite magnetic polarities appear on the walls of the defect cylinder creating a dipole. ${ }^{12}$ Because the defect is small in comparison to the region of the sample under the action of a uniform magnetic field this charge was assumed to be distributed homogeneously on the surface of the defect as shown in Figure 3.

Half of the cylindrical defect develops a north polarity or positive magnetic charge density, $+\sigma$, while the other half has a south polarity or negative magnetic charge density, $-\sigma$. The cylindrical defect has a radius of $R_{1}$. The angle $\theta_{1}$ is measured from the positive $y$ direction to an element of magnetic charge, $d p_{1}$. The differential element of charge at the defect, $d p_{1}$, has coordinates $\left(R_{1} \sin \theta_{1}, R_{1} \cos \theta_{1}, z_{1}\right)$ and a charge proportional to its area.

The magnetic field $\left(d H_{1}\right)$ generated at a distance $r_{1}$ by this element of charge $d p_{1}$ is given by ${ }^{11}$

$$
d H_{1}=\frac{d p}{4 \pi r_{1}^{3}} \cdot \mathbf{r}_{\mathbf{1}} .
$$

The $z$ coordinate of the observation point represents the lift-off of the detection Hall probe $h$, and it is always a positive constant since we perform scans at a constant level. Initially, only the positive polarity side $\mathrm{H}^{+}$of each defect and $\theta_{1}$ measured from the positive $y$ axis in a clockwise direction is considered.

The components of the field at a distance $r_{1+}$ are given by, 


$$
\begin{gathered}
d H_{z}^{+}=\frac{\sigma R_{1} d \theta_{1} d z_{1}}{4 \pi r_{1}^{3}}\left(h-z_{1}\right) \\
d H_{x}^{+}=\frac{\sigma R_{1} d \theta_{1} d z_{1}}{4 \pi r_{1}^{3}}\left(x+R_{1} \sin \theta_{1}\right) .
\end{gathered}
$$

The $y$ component of the leakage field, $d H_{y}^{+}$, vanishes due to the symmetry. An integration of Equation 2 is applied over $\theta_{1}$ from 0 to $\pi$ and over $z$ from $-b 1(b>0)$ to 0 to determine the total field at $r_{1+}$ due to the positively polarized side of the defect:

$$
H_{z}^{+}=\int_{0}^{\pi} d \theta_{1} \times \int_{-b 1}^{0} \frac{\sigma R_{1}\left(h-z_{1}\right) d z_{1}}{4 \pi\left\{\left(x+R_{i} \sin \theta_{1}\right)^{2}+\left(R_{i} \cos \theta_{1}\right)^{2}+\left(h-z_{1}\right)^{2}\right\}^{\frac{3}{2}}}
$$

By using the same approach for the negatively polarized side $H_{z}^{-}$of the cylinder. The total normal leakage field along the $x=0$ profile is given by,

$$
d H_{z}=d H_{z}^{+}+d H_{z}^{-} .
$$

Each of these signal components are measured by hall probes and used in the analysis process. Finite element analysis in computer based simulation software calculates these values using a numerical approach which becomes more complex when it comes to multiple defects.

\subsection{Simulation with Finite Element Analysis}

Our approach highly relies on extensive data available to learn the models. It is obvious that collecting measurements by introducing various defect dimensions using a real tool is exhaustive and prohibitive. That is why we have chosen to use computer based simulation software to simulate the physical scenarios. Following this, a simulation model of the MFL setup (see Figure 1) was created using the AC/DC module within COMSOL Multiphysics software.

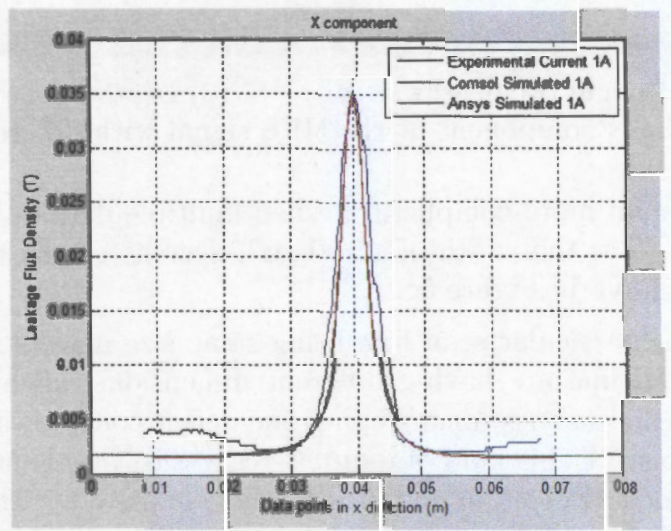

Figure 4: Comparison between COMSOL, ANSYS and the experimental setup.

Since a 2-D model could only account for highly symmetric flaws, a 3-D model simulating the real MFL setup tool was designed. Two coils were wound in the two legs of a U shaped yoke made of soft iron. The two coils are supplied with a DC supply in-order to magnetize the test substance. The measurements are taken in a plane $2 \mathrm{~mm}$ above and parallel to the test plate. By defining a fixed sensor array, the repeatability of the simulated measurements is achieved. A very dense mesh, consisting 122686 elements through out the model was used in the FEA. The solution was extracted when the "relative tolerance" of each consecutive iteration goes below $0.1 \%$. In order to validate the simulation model, a similar exercise was carried out in ANSYS software giving a $98.84 \%$ agreement in the signals. Figure 4 shows a comparison between the outputs of these two FEA packages, together with the output of the actual MFL experimental setup. 
Let us consider a scenario with a single defect appeared in one particular scan. Dimensions of this defect is iteratively changed in the FEA simulation while capturing the MFL signals. The values of these configurations are given in the single defect row of the Table 1 . The bottom of the defect for each depth value is considered flat and the walls of the defects are assumed perpendicular to the bottom surface. An example of the MFL signals for a given horizontal line when the depth of the defect is changed, keeping all other parameters the same is shown in Figure 5a. This figure shows that when the defect depth increases, the amplitude of the X component increases. However, that is not the only reason for this to happen; when the diameter of the circular defect is increased, keeping all other parameters the same, the width of the signal increases along with the magnitude as illustrated in Figure 5b.

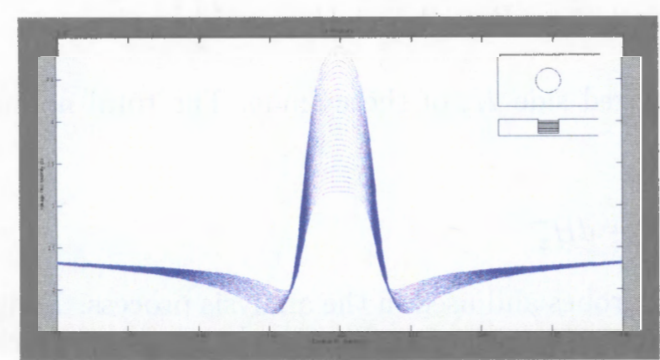

(a) Varying the depth of one defect

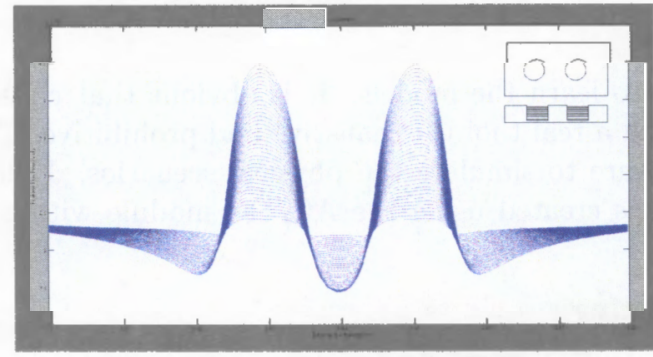

(c) Varying the depth of two defects

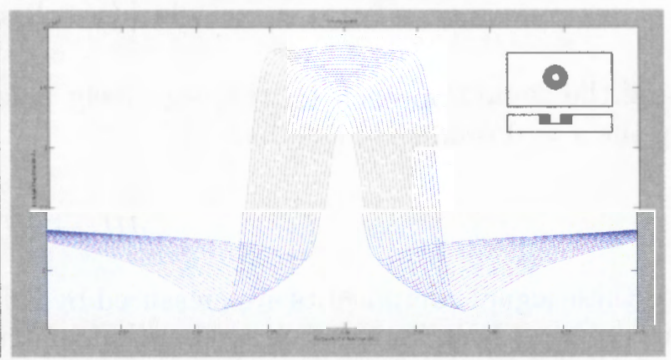

(b) Varying the width of one defect

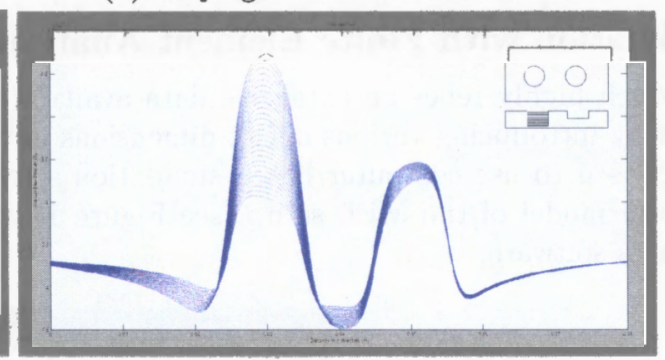

(d) Depth of one defect out of two

Figure 5: Behavior of the $\mathrm{X}$ component of the MFL signal with different defect configurations.

These relationships become even more complicated when multiple defects happen to be present in the scan line. Measured magnitude, as well as the width of the signal, decreases with the number of defects while their dimensions remain constant as shown in Figure 5c.

All the defects present in one particular scan line being same size defects is a very unrealistic scenario. So when the two defects in the scan line are having different dimensions, all above relationships become much more complex. Figure 5d shows an analysis done keeping one defect constant and other one changing its depth. Machine learning can be used to solve this kind of complex regression problems. For instance, A.A. Carvalho et al ${ }^{8}$ used artificial neural networks for detection and classification of defects without characterising the size of the defects. The main drawback of using ANN is that it does not give any confidence indication on the prediction, i.e. there is no measure of uncertainty. This paper takes a Bayesian view of the problem, maintaining a posterior over interpolants rather than simply the maximum-likelihood interpolant, giving a measure of uncertainty in the geometric characterisation of the defects. In this work the proposition is to accomplish this within the Gaussian process framework. ${ }^{13}$

\section{FEATURE EXTRACTION}

Although feature extraction from MFL signals is not the main focus of this paper, we provide a brief discussion of the features used. First, we extract some straightforward features from the MFL signals to be used in the learning 
process, namely for the $X$ component, heights and width of each peak, distances between peaks, and number of peaks (Figure 6a illustrates these basic features for $X$ component). For the $Y$ component, as illustrated in Figure $6 \mathrm{~b}$, height differences of each parts of the signal as well as the distances between peaks and valleys were used. The main reason for using these features is that we noticed significant changes of these features with respect to the various depths and widths in our preliminary work with simple defect configurations.

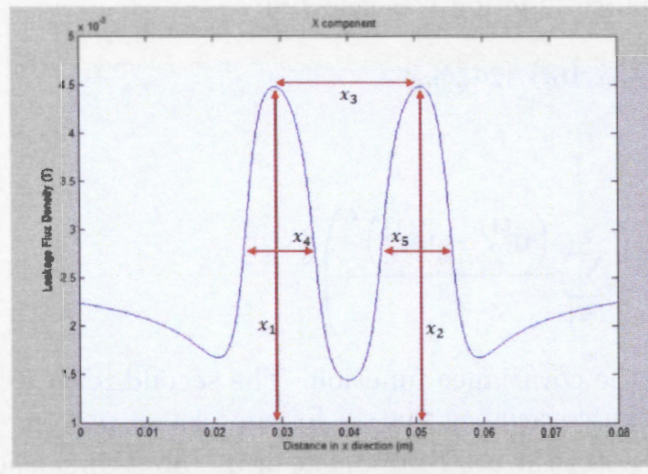

(a) Extracted features from the $X$ component

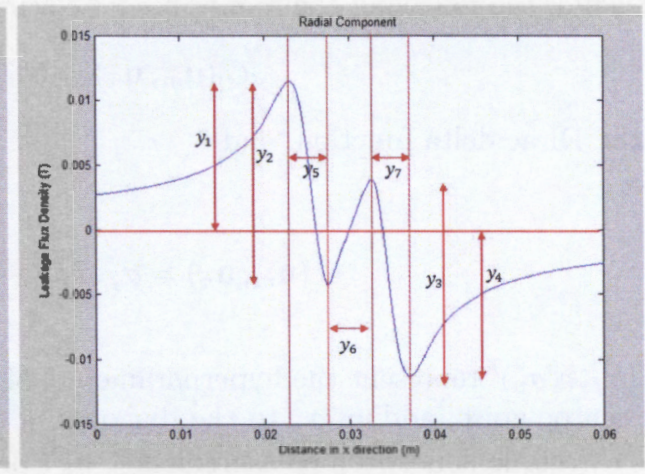

(b) Extracted features from the $Y$ component are being used in the modeling process.

Later, we extended this set of features with the Hjorth Transform ${ }^{14}$ parameters, which extract statistical information about the MFL signals. The Hjorth Transform method takes into account the standard deviation, the standard deviations of the amplitude and the second derivative. From these standard deviations a set of parameters are derived. These parameters are: 1. Activity, giving a measure of the squared standard deviation of the amplitude, sometimes referred to as the variance or mean power. 2. Mobility, giving a measure of the standard deviation of the slope with reference to the standard deviation of the amplitude. It is expressed as a ratio per time unit and may be conceived also as a mean frequency. 3. Complexity, giving a measure of excessive details with reference to the "softest" possible curve shape, the sine wave, this corresponding to unity. It is expressed as the number of standard slopes actually generated during the average time required for generation of one standard amplitude as given by the mobility. Due to the non-linear calculation of standard deviation this parameter will quantify any deviation from the sine shape as an increase from unity. These three parameters will be used as features of the MFL signals.

All these features form the low-dimensional input vectors $\mathbf{u}_{i}$ of the data set $\Psi$.

\section{BAYESIAN LEARNING WITH GP}

Gaussian Processes are a non-parametric tool in the sense that they do not explicitly specify a functional model between inputs and outputs. GP can be thought of as a Gaussian prior over the function space mapping inputs and outputs. ${ }^{1}$

The problem of learning a MFL signal is one of regression: estimating a function $f$ mapping from inputs $\mathbf{u}$ to output $v=f(\mathbf{u})$. In the context of the present problem, the outputs are the geometrical characteristics of the defects, i.e. depth, width, separation and number of defects, and the inputs are the features of MFL signals. To apply a GP framework to this regression problem, one must first select a hyperfunction, called a covariance function. This specifies the kinds of functions that are expected, before any data have been seen. Technically, the covariance function places a prior likelihood on all possible functions. Next, the hyperparameters of the covariance function are learned from the training data. Finally, the combination of the training data and the covariance function induces not only the most likely defect dimension, but also a full posterior probability distribution over all possible defect dimensions.

More formally, we are given a set of $\mathrm{N}$ noisy measurements $\mathbf{u}_{1, \ldots, N}$ associated with the outputs $v_{1, \ldots, N}$. The defining characteristic of a GP is that for any finite set of points $\mathbf{u}_{1, \ldots, N}$, the marginal density $p\left(f\left(\mathbf{u}_{1}\right)\right.$, 
$\left.f\left(\mathbf{u}_{2}\right), \ldots, f\left(\mathbf{u}_{N}\right)\right)$ is a multivariate Gaussian. A Gaussian process is completely specified by its mean function $m(\mathbf{u})=E[f(\mathbf{u})]$ and its covariance function $\left.C\left(\mathbf{u}, \mathbf{u}^{\prime}\right)\right)=E\left[(f(\mathbf{u})-m(\mathbf{u}))\left(f\left(\mathbf{u}^{\prime}\right)-m\left(\mathbf{u}^{\prime}\right)\right)\right]$ as $f(\mathbf{u}) \sim$ $\mathcal{G P}\left(m(\mathbf{u}), C\left(\mathbf{u}, \mathbf{u}^{\prime}\right)\right)$. The first step is to choose the form of $C\left(\mathbf{u}, \mathbf{u}^{\prime}\right)$, which specifies the covariance of this Gaussian for any pair of points. The choice of covariance function is a form of model selection for automatic relevance determination, and should be consistent with prior knowledge about the type of function expected. For the problem at hand, the following commonly used ${ }^{15}$ covariance function was selected:

$$
C\left(\mathbf{u}_{m}, \mathbf{u}_{n}\right)=C_{i}\left(\mathbf{u}_{m}, \mathbf{u}_{n}\right)+\sigma_{v}^{2} \delta_{m n}
$$

where $\delta$ is the Dirac delta function, and

$$
C\left(\mathbf{u}_{m}, \mathbf{u}_{n}\right)=\sigma_{f} \exp \left(-\frac{1}{2} \sum_{l=1}^{L} \frac{\left(\mathbf{u}_{m}^{(1)}-\mathbf{u}_{n}^{(1)}\right)^{2}}{r^{2}}\right)
$$

where $\beta=\left(\sigma_{f}, r, \sigma_{v}\right)^{\prime}$ represent the hyperparameters of the covariance function. The second term in Equation 6 defines the sensor noise, adding $\sigma_{v}^{2}$ to the diagonal of the covariance matrix for any set of points. Equation 7 encodes the correlations between measurements. $\mathbf{u}_{n}^{(d)}$ denotes the $d_{t h}$ component of $\mathbf{u}_{n}$, an $d$-dimensional vector, i.e. the dimension of the feature space.

\subsection{Hyperparameters of the Covariance Function}

Multidimensional outputs are problematic for GPs. ${ }^{15}$ In this study, we treat each dimension of the defect independently, building a separate model (with separate covariance function hyperparameters) for each of them. This is an approximation implying no-correlation between the different dimensions of the multiple defects.

In a Bayesian framework, determining the hyperparameters involves first specifying a prior belief about the values of the parameters. Uncertain priors were chosen for all hyperparameters except the sensor noise $\sigma_{v}$, since it can be experimentally characterised.

The next step is to learn the hyperparameters from the training data. Ideally, we would integrate over all possible values of the parameters to estimate the posterior distribution $p(\beta \mid \Psi, C()$.$) , where C($.$) denotes the$ form of the covariance function. However, since this is analytically intractable, we make the assumption that $p(\beta \mid \Psi, C()$.$) is sharply peaked around the most probable values, and approximate the integral by using these$ values. The most probable values can be found using gradient descent. The posterior over the hyperparameters is given by

$$
\left.p(\beta \mid \Psi, C(.)) \propto P\left(V_{N} \mid U_{1, \ldots, N}, C(.), \beta\right) p(\beta)\right),
$$

where $V_{N}$ is a vector of training noisy outputs. Let $C_{N}$ denote the covariance matrix relating the $\mathrm{N}$ outputs. The $\log$ likelihood $L=\log \left(p\left(v_{N} \mid \mathbf{u}_{1, \ldots, N}, C(),. \beta\right)\right) p(\beta)$ is given by,

$$
L=-\frac{1}{2} \log \left(\operatorname{det} C^{N}\right)-\frac{1}{2} v_{N}^{T} C_{N}^{-1} v_{N}-\frac{N}{2} \log 2 \pi
$$

and the gradient of the log-likelihood is given by

$$
\frac{\partial L}{\partial \beta}=-\frac{1}{2} \operatorname{trace}\left(C_{N}^{-1} \frac{\partial C_{N}}{\partial \beta}\right)+\frac{1}{2} v_{N}^{T} C_{N}^{-1} \frac{\partial C_{N}}{\partial \beta} C_{N}^{-1} v_{N}
$$

as shown by Gibbs and MacKay. ${ }^{16}$

This process allows all hyperparameters to be learned simultaneously from the data. The learning process implicitly trades off the various parameters such as sensor noise and the features' ability to generalise spatially. Note that there are relatively few free parameters that need to be tuned by hand: only the choice of the covariance function and the priors over $\beta$. A disadvantage of this process is that it is relatively computationally intensive, since each step of gradient descent requires the inversion of the $N \times N$ matrix $C_{N}$. While exact methods were sufficient for the data sets addressed in this paper, Rasmussen and Williams describe a number of approximate methods. ${ }^{1}$ 


\section{RESULTS}

\subsection{Data sets}

A soft iron plate was simulated using different defect configurations and MFL signals were captured in each scenario. Table 1 shows the different defect configurations used. Figure 5 shows graphical examples of some of these simulations.

Table 1: Different defect sizes and configurations used in the training process.

\begin{tabular}{|c|c|c|c|}
\hline Defect & Depth & Width (Diameter) & Separation between centers \\
\hline Single & $\begin{array}{l}0.5 \mathrm{~mm} \text { to } 4.5 \mathrm{~mm} \\
\text { with a step of } \\
0.25 \mathrm{~mm}\end{array}$ & $\begin{array}{l}5 \mathrm{~mm} \text { to } 25 \mathrm{~mm} \text { with a } \\
\text { step of } 1 \mathrm{~mm}\end{array}$ & \\
\hline Two Circular & $\begin{array}{l}0.5 \mathrm{~mm} \text { to } 4.5 \mathrm{~mm} \\
\text { with a step of } \\
0.25 \mathrm{~mm}\end{array}$ & $\begin{array}{l}5 \mathrm{~mm} \text { to } 25 \mathrm{~mm} \text { with a } \\
\text { step of } 1 \mathrm{~mm}\end{array}$ & $\begin{array}{l}3 \mathrm{~mm} \text { to } 20 \mathrm{~mm} \text { with a step of } 1 \\
\mathrm{~mm}\end{array}$ \\
\hline Rectangular & $\begin{array}{l}0.5 \mathrm{~mm} \text { to } 4 \mathrm{~mm} \\
\text { with a step of } \\
0.0175 \mathrm{~mm}\end{array}$ & $\begin{array}{l}2 \mathrm{~mm} \text { to } 20 \mathrm{~mm} \text { with a } \\
\text { step of } 0.25 \mathrm{~mm}\end{array}$ & \\
\hline
\end{tabular}

We apply a magnetic field of $1.25 * 10^{6} \mathrm{~A} / \mathrm{m}$ keeping South and North poles in a distance of $23 \mathrm{~cm}$. We selected all these numbers to fit the experimental setup we developed to validate the simulation model (see Figure 4). Although we use two electromagnets in the experimental setup, in simulation we use two permanent magnets providing the same magnetic field. By doing this we could reduce the degrees of freedom (DOF) of each FEA simulation to 216531 which is a $20 \%$ " improvement in terms of DOF and about 45 minutes saving in terms of time per simulation. Note that each curve in the results, for instance a single curve in Figure 5 , corresponds to a single simulation.

\subsection{Machine Learning}

For learning and evaluating the inverse MFL model, the ground truth is essential as mentioned in Section 3. Since the simulation scenarios we present here are well defined geometrical shapes, numerical algorithms are used to generate the ground truth for each scenario.

Separate models have been learned to infer each dimension of the defect configuration independently; e.g. depth, width and separation. The data sets described in Table 1 correspond to $\Psi=\left\{\left(\mathbf{u}_{0}, v_{0}\right),\left(\mathbf{u}_{1}, v_{1}\right), \ldots,\left(\mathbf{u}_{N}, v_{N}\right)\right\}$, where $v_{i}$ represents either, depth, width or separation in the modeling process. For instance in case of the depth, these data aim to cover various defect configuration for each depth profile.

After the training process the mode and the hyperparameters of the covariance function are learned. An example of the resulting variation of the one of the features, namely the magnitude of the $\mathrm{X}$ component, with respect to the depth is illustrated in Figure 7a. The model becomes more complex when all the configurations come into play as described in Section 4.1, so the variation of other the depth with respect other features are not displayed to avoid confusion.

Once the model has been learned, it is used to infer the defect parameters from fresh measurements. Note that to validate the performance of our approach, we use a 3-fold cross-validation method.

The same exercise has been employed to predict the width, separation and the location of the defect as well, producing prominent results as shown in Table 2. Although the location is a straight forward problem, having a linear relationship with the peak location of the $\mathrm{X}$ component of the leakage field, we stick into our framework, using GP models to solve the location as well. Figure $7 \mathrm{~b}$ illustrates the comparison between the real and the predicted values. To generate the Figure $7 \mathrm{~b}$ only the mean prediction values have been used. But one of the main advantages of a GP model is that the model produces an uncertainty indication with each prediction. So the 


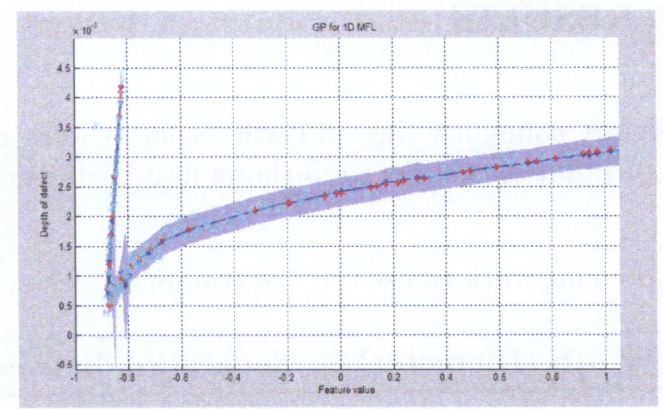

(a) Magnitude of the $\mathrm{X}$ component variation with the defect depth

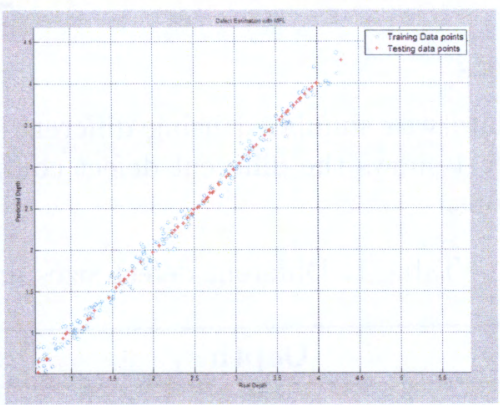

(b) Comparison between the predicted and actual depth

Figure 7: Learning and evaluation of results of the GP model.

Table 2: Mean squared error values for each GP model.

\begin{tabular}{cc}
\hline Model & Mean squared error \\
\hline Depth of the defect & 0.000824 \\
Width of the defect & 0.003417 \\
Defect separation & 0.000148 \\
\hline
\end{tabular}

end result comes with bounds on the certainty of the prediction. We use mean square as a measure to evaluate the models. Since each of defect dimension has its own model, the mean square error for each model is indicated in Table 2.

\subsection{Experiments}

In order to validate our approach, we have taken real measurements with the MFL experimental setup of the soft plate shown in Figure 8a. A L12 series Firgelli Miniature Linear slider was used to slide a single hall sensor along the $X$ axis of the plate. An ARDUINO UNO development board was used to drive the slider, as well as, to acquire the signal from the sensor. We have extracted the features presented in Section 5 from the MFL signals and use them as an input of the GP models. A 3-D reconstruction of the plate is presented in Figure $8 \mathrm{~b}$. Top and side views of this reconstruction are shown in Figures $8 \mathrm{c}, 8 \mathrm{~d}$ and $8 \mathrm{e}$.

When it comes to the stress analysis and lifetime predictions it is of immense importance to have the profile of the anomalies of a particular metal section. Although it is not the focus of this paper, in favor of that, all the developed models have been combined together to generate a complete 3 dimensional profile of the defects (Figure 8b). Moreover Table 3 shows the numerical comparison of the actual and predicted defect parameters. Not only the model prediction error but also various other experimental errors contribute to the accuracy of the results. For example the linear slider which was used to slide the sensor along with the mechanical arrangement has $\sim \pm 0.5 \mathrm{~mm}$ error margin. Considering all these sources of noise, the GP modeling framework introduced in this paper can be successfully used to interpret MFL signals in practical scenarios.

Table 3: Numerical comparison of real and predicted parameters of defects.

\begin{tabular}{ccccc}
\hline & \multicolumn{2}{c}{ Left Defect } & \multicolumn{2}{c}{ Right Defect } \\
\hline & Real & Reconstructed & Real & Reconstructed \\
Diameter (maximum width) & $10 \mathrm{~mm}$ & $11.3 \mathrm{~mm}$ & $20 \mathrm{~mm}$ & $19.1 \mathrm{~mm}$ \\
Average Depth & $2.5 \mathrm{~mm}$ & $2.375 \mathrm{~mm}$ & $2.5 \mathrm{~mm}$ & $2.325 \mathrm{~mm}$ \\
\hline & Real & Reconstructed & & \\
Defect Separation & $25 \mathrm{~mm}$ & $26.1 \mathrm{~mm}$ & & \\
\hline
\end{tabular}




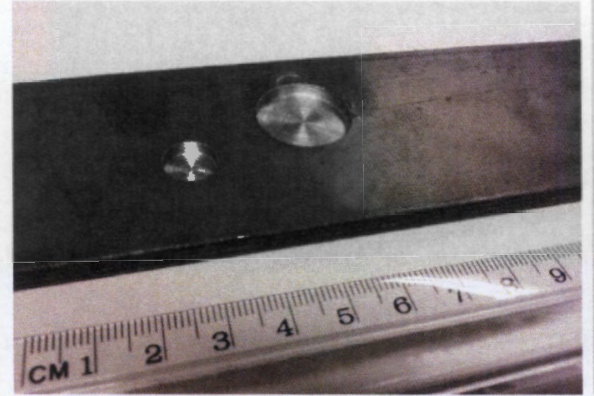

(a) Soft iron plate used in the experiment.

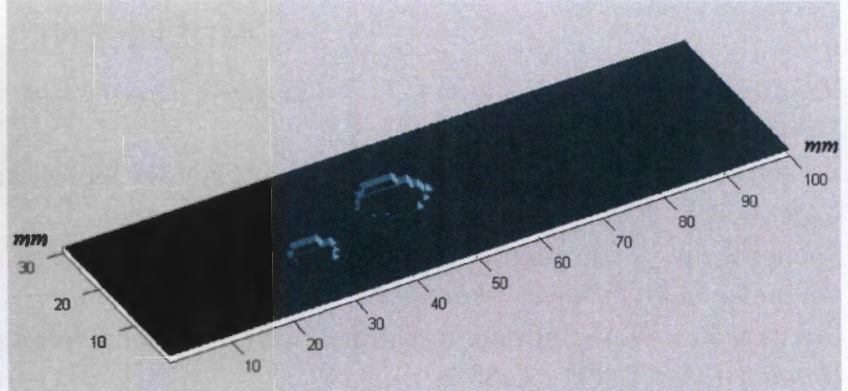

(b) $3 \mathrm{D}$ reconstructed plate.

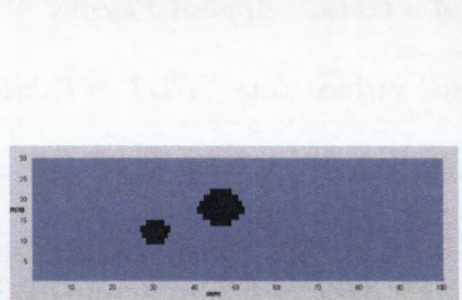

(c) Top view of the reconstruction.

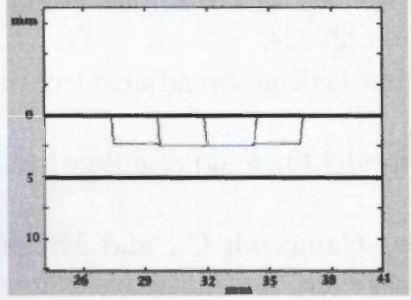

(d) Side view of left defect.

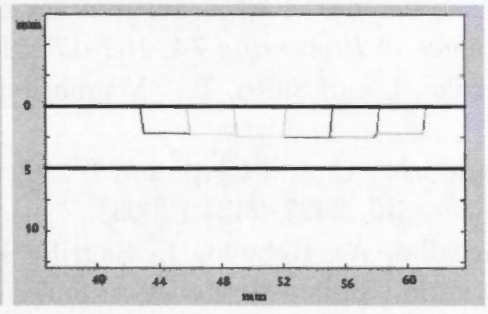

(e) Side view of right defect.

Figure 8: Comparison of the defect depth profile.

\section{CONCLUSIONS}

In this study we have proposed an approach to solve the inverse model problem of defect detection and sizing for MFL signals by employing Gaussian process regression.

A FEA based simulation model was exploited to generate sufficient amount of data needed for the training process of the proposed supervised learning algorithm. The soundness of the simulation model was validated prior to the data generation process by comparing its signals with an experimentally implemented MFL setup.

Both circular as well as rectangular shaped defects were used in the training process in order to infer any irregular shape. The developed GP model was used to infer multiple defects dimensions, which were in turn compared qualitatively and quantitatively with the ground truth showing correct agreement.

The clear advantage of the method presented here is that the obtained model is nonparametric with only few parameters are manually tuned. Moreover each prediction come along with the uncertainty values associated with it; the more data use for the training process, the tighter uncertainty margins of the prediction.

In the current approach, although the signals are captured using a 2-D array of sensors, only 1-D samples of the signal is extracted as the actual measurement, i.e. 1-D scan line. In the future, we are planning to use the 2-D signal itself in the modelling process. Features to describe this 2-D signal of the leakage field need to be investigated.

\section{ACKNOWLEDGMENTS}

This publication is an outcome from the Critical Pipes Project funded by Sydney Water Corporation, Water Research Foundation of the USA, Melbourne Water, Water Corporation (WA), UK Water Industry Research Ltd, South Australia Water Corporation, South East Water, Hunter Water Corporation, City West Water, Monash University, University of Technology Sydney and University of Newcastle. The research partners are Monash University (lead), University of Technology Sydney and University of Newcastle. 


\section{REFERENCES}

[1] Rasmussen, C. and Williams, C., [Gaussian Process for Machine Learning], MA:MIT press, Cambridge (2006).

[2] Zatsepin, N. N. and Shcherbinin, V. E., "Calculation of the magnetostatic field of surface defects," Defectoskopiya 5, 50-59 (1966).

[3] Shcherbinin, V. E. and Pashagin, A. I., "Influence of the extension of adefect on the magnitude of its magnetic field," Defectoskopiya 8, 74-82 (1972).

[4] Förster, F., "New findings in the field of non-destructive magnetic leakage field inspection," NDT International 19, 3-14 (Feb. 1986).

[5] Minkov, D., Takeda, Y., Shoji, T., and Lee, J., "Estimating the sizes of surface cracks based on Hall element measurements of the leakage magnetic field and a dipole model of a crack," Applied Physics A: Materials Science \& Processing 74, 169-176 (Feb. 2002).

[6] Uetake, I. and Saito, T., "Magnetic flux leakage by adjacent parallel surface slots," NDT $\&$ E International

[7] Mandache, C. and Clapham, L., "A model for magnetic flux leakage signal," Journal of Physics D: Applied Physics 36, 2427-2431 (2003).

[8] Carvalho, A., Rebello, J., Sagrilo, L., Camerini, C., and Miranda, I., "MFL signals and artificial neural networks applied to detection and classification of pipe weld defects," NDT \& E International 39, 661-667 (Dec. 2006).

[9] Ramuhalli, P., Udpa, L., and Udpa, S. S., "Neural network-based inversion algorithms in magnetic flux leakage nondestructive evaluation," Journal of Applied Physics 93(10), 8274-8276 (2003).

[10] Brooks, A., Makarenko, A., and Upcroft, B., "Gaussian Process Models for Indoor and Outdoor SensorCentric Robot Localization," IEEE Transactions on Robotics 24, 1341-1351 (Dec. 2008).

[11] Edwards, C. and Palmer, S. B., "The magnetic leakage field of surface-breaking cracks," Journal of Physics D: Applied Physics 19(4), 657 (1986).

[12] Dutta, S., Ghorbel, F., and Stanley, R., "Dipole Modeling of Magnetic Flux Leakage," IEEE Transactions on Magnetics 45, 1959-1965 (Apr. 2009).

[13] Williams, C. K. I. and Rasmussen, C. E., "Gaussian processes for regression," in [Advances in Neural Information Processing Systems 8], 514-520, MIT Press (June 1996).

[14] Hjorth, B. O., "Technical contributions eeg analysis based on time domain properties," Electroncephalography and Clinical Neurophysiology 29, 306-310 (1970).

[15] MacKay, D., "Introduction to gaussian processes," NATO ASI Series F Computer and Systems Sciences 168, 133-166 (1998).

[16] Gibbs, M. and MacKay, D., "Efficient implementation of gaussian processes," tech. rep., Cavendish Lab., Cambridge, UK (1997). 